\title{
OSTEOFIBROUS DYSPLASIA OF THE ULNA
}

\author{
S. Kamineni, T. W. R. Briggs, A. Saifuddin, A. Sandison \\ From the Royal National Orthopaedic Hospital NHS Trust, Stanmore and the London \\ Bone and Soft-Tissue Tumour Service, London, England
}

O steofibrous dysplasia is a rare condition usually affecting the tibiae and fibulae of males in the first two decades of life. Involvement at the ulna has also been reported in some cases. We describe an extensive, rapidly progressive lesion of the ulna in a three-year-old girl. The progression of the disease accelerated after a local marginal removal, and required extensive resection of the ulna with reconstruction using a fibular autograft.

The particular features of this case are the change in the aggressive nature of the condition, which has not been previously reported. We believe that this is only the second recorded case of histologically proven involvement of the ulna.

J Bone Joint Surg [Br] 2001;83-B:1178-80.

Received 31 January 2001; Accepted 20 March 2001

Osteofibrous dysplasia was first described as congenital osteitis fibrosa by Frangenheim. ${ }^{1}$ Kempson ${ }^{2}$ later noted lesions resembling fibrous dysplasia, termed 'ossifying fibroma', in the tibiae of two young children which were histologically distinguished by the presence of active osteoblasts on abnormal bone trabeculae. The nomenclature was further confused when the condition was referred to as 'congenital pseudoarthrosis', 3 and 'congenital fibrous defect' ${ }^{4}$ Campanacci ${ }^{5}$ first described the lesion as 'osteofibrous dysplasia of the tibia and fibula', thereby emphasising its histological similarity to fibrous dysplasia, and its commonest anatomical locations. Most reported cases involve the tibia or the fibula; ${ }^{5,6}$ involvement of the ulna is very uncommon. ${ }^{7,8}$ There is no consensus as to the best management of this condition in the tibia, and only two previous reports in the English literature have described involvement of the ulna. ${ }^{7,8}$ We present a case of aggressive osteofibrous dysplasia of the ulna.

\section{Case report}

A three-year-old girl fell sustaining a fracture of the midshaft of the ulna, through a small cystic lesion. This was successfully managed conservatively in a plaster cast. One year later, however, there was local tenderness without loss of function. Radiographs revealed an enlargement of the original lesion, which had become expansile with surrounding cortical thickening. The remainder of the lesion showed a ground-glass matrix, with areas of poor margination and reactive sclerosis (Fig. 1).

After biopsy with a Jamshidi needle, histological examination showed fibrous dysplasia, and she was therefore managed conservatively. Three months later the dorsal swelling of the ulna had enlarged. Radiographs confirmed a further increase in the size of the lesion. An open 'shark-bite' resection was carried out using a dorsal approach, and the lesion was resected to its macroscopic margins. The resected material showed features of osteofibrous dysplasia (Fig. 2). Over the next four months the lesion progressed rapidly to involve most of the remaining ulna (Fig. 3). The forearm was more painful and tender, and there was posterolateral bowing. Because of the progression and extent of the pathological process, further excision was carried out.

Under general anaesthesia, $15 \mathrm{~cm}$ of the shaft of the ipsilateral fibula was harvested. The ulna was approached through the posterior subcutaneous border and $12 \mathrm{~cm}$ were resected under fluoroscopic guidance. Histological examination of an imprint

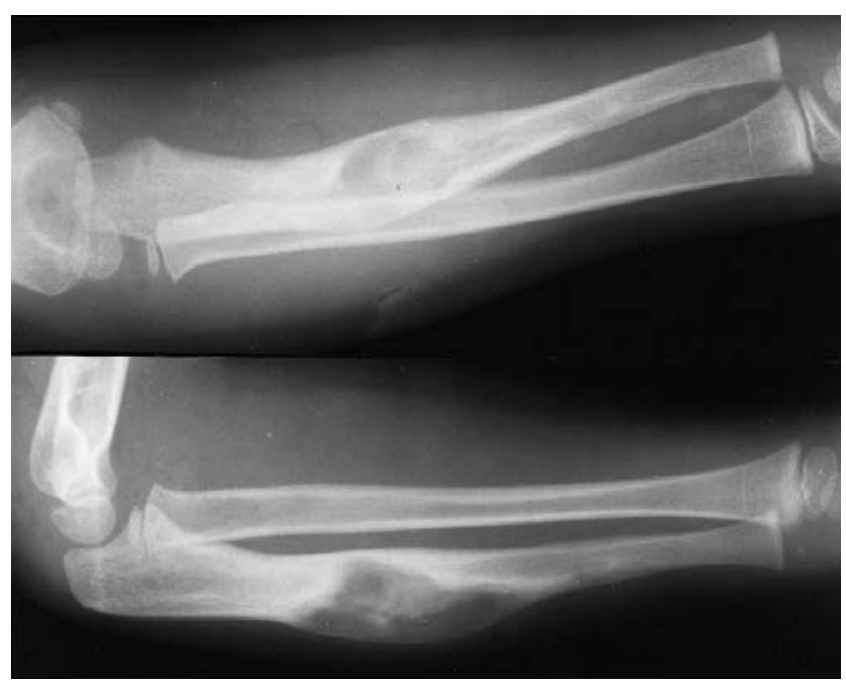

Fig. 1

Anteroposterior and lateral radiographs of the ulna showing an expanded lesion in the posterior cortex of the diaphysis. It has a ground-glass matrix and reactive sclerosis in the adjacent medulla. 


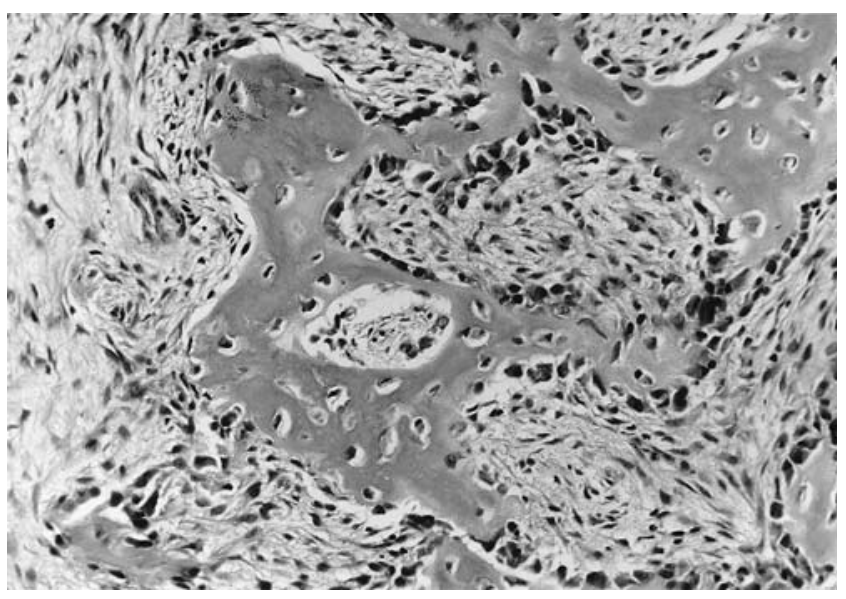

Fig. 2

Biopsy specimen photographed at medium power showing osteofibrous dysplasia. There are irregular trabeculae of woven bone with a layer of osteoblasts on the surface. There is a proliferation of bland spindle cells between the bony trabeculae (haematoxylin and eosin $\times 10$ ).

from tissue at the proximal margin revealed an area of suspicion, requiring the removal of a further $3 \mathrm{~mm}$ of the proximal ulna. The fibular graft was fashioned into a barb and inserted into the proximal ulnar remnant, with firm fixation. The distal aspect of the graft was trimmed to allow apposition and to maintain correct ulnar variance under fluoroscopic control; it was fixed by a twohole semitubular plate. The range of forearm rotation was equal to that of the normal contralateral side. The forearm was protected in an above-elbow plaster cast for ten weeks. After six months, there was a full range of rotation of the forearm, although there was minor angulation at the proximal junction of the graft. Radiographs revealed satisfactory bony union (Fig. 4).

\section{Discussion}

Campanacci's description of osteofibrous dysplasia of the tibia and fibula highlighted the characteristic pathology and its pre-
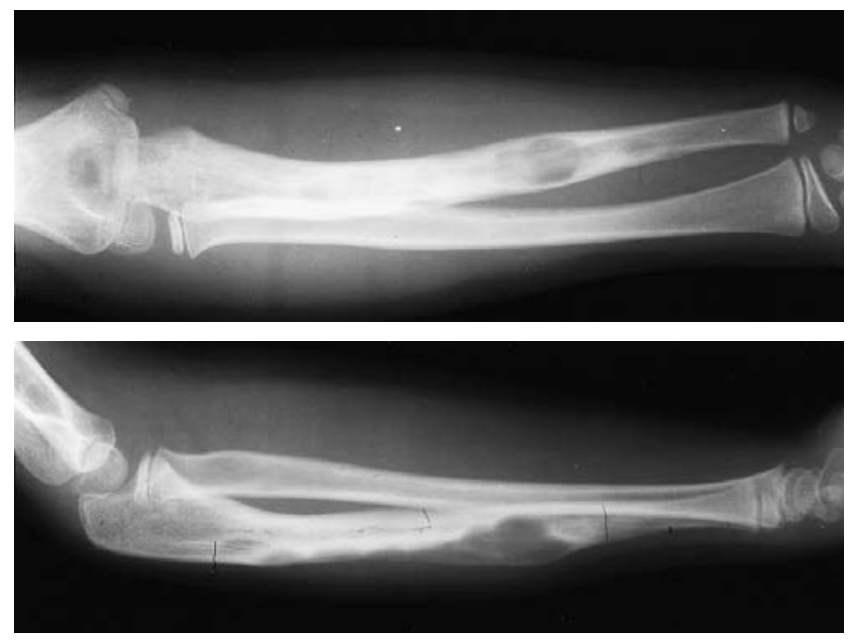

Fig. 3

Anteroposterior and lateral radiographs of the ulna showing extension of the pathological process, both proximally and distally. dilection for these two bones. ${ }^{9}$ Much of the knowledge regarding its natural history and management is from such cases. As demonstrated by this and other reports, ${ }^{7,8}$ the ulna is another recognised, but rare, site with the midshaft, as for the tibia, most often involved. Only one previous case of ulnar osteofibrous dysplasia has been described with a convincing histological diagnosis. ${ }^{8} \mathrm{~A}$ bilateral case has also been reported, without supporting histological assessment. ${ }^{7}$ In the first patient, multiple recurrences followed curettage and grafting procedures and in the second spontaneous resolution occurred.

Osteofibrous dysplasia appears to have a male predominance, and symptoms tend to occur in the first two decades of life. The most common presenting complaint is of swelling or deformity, with a typically painless bowing of the involved segment of the limb. ${ }^{5,10}$ Pathological fracture may be the presenting feature. Another significant complication, described in 1989, is the coexistence of an adamantinoma within an osteofibrous dysplasia-like lesion, ${ }^{11}$ but the relationship between these two conditions is unclear.

Radiologically, osteofibrous dysplasia has either a typical eccentric or intracortical location and either confluent multiloculation with an expanded and thinned overlying cortex, or osteolysis with a surrounding sclerotic rim. ${ }^{5,8}$ The lesion is often well marginated with an occasional ground-glass appearance, but there is neither a periosteal reaction nor an associated soft-tissue swelling. The main differential diagnoses are fibrous dysplasia, adamantinoma, and non-ossifying fibroma, but may also include eosinophilic granuloma, Ewing's sarcoma, vascular tumours, and osteomyelitis. $5,6,12$

It has previously been considered that the diagnosis could be made on the basis of the characteristic clinical and radiological features. ${ }^{9}$ Since the discovery of coexistent epithelioid tumours ${ }^{12}$ it is now mandatory to have histological confirmation of the diagnosis. ${ }^{13}$ There are two characteristic histological features. First, there are active fibroblasts bordering bony trabeculae embedded in a fibrous stroma, with each trabecula lined by active osteoblasts, and, secondly, a centrifugal zonal architecture, with peripheral lamellar bony trabeculae, merging with the overlying cortex, and central scant woven trabeculae in a mainly fibrous stroma. These latter features help to distinguish this lesion from its closest differential diagnosis, fibrous dysplasia. ${ }^{9}$ The pitfalls of
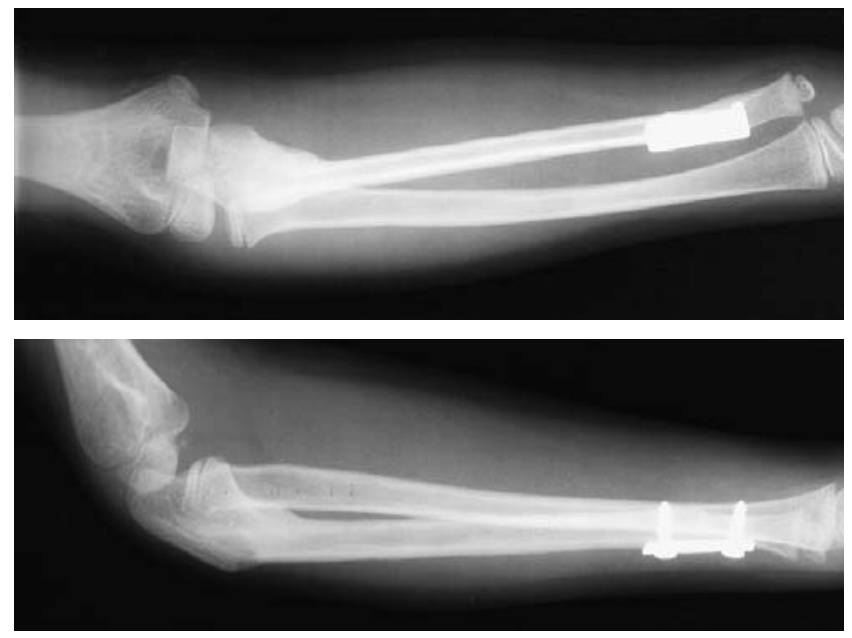

Fig. 4

Anteroposterior and lateral radiographs of the ulna showing bony union of the fibular graft. 
relying on a histological diagnosis are exemplified in this case, and Park et $\mathrm{al}^{6}$ demonstrated that cytokeratin immunostaining may be unreliable, with poor sensitivity. Our recommendation is that biopsies should be taken from multiple areas of suspicion and a histopathologist specialising in the pathology of bone tumours should make the final diagnosis.

The natural history of these lesions is usually of gradual growth during the first decade of life, with stabilisation by the age of 15 years. ' Less common patterns include benign, non-aggressive lesions which may regress spontaneously, and aggressive lesions which may involve the whole bone, with resultant bowing. The management has ranged from simple immobilisation to surgical resection. Successful conservative management for ulnar lesions has been reported, with spontaneous resolution by the age of 12 years. ${ }^{7}$ Patients under the age of 15 years treated by curettage and subperiosteal and extraperiosteal resection often have local recurrence. ${ }^{5,8}$ Rates of recurrence ranging from $69 \%$ to $100 \%$ have been reported after treatment by curettage and bone grafting. $9,10,13,14$ These figures decrease significantly when surgical treatment is instigated after the age of ten years, and recurrence is extremely rare after the age of 15 years. ${ }^{9}$ Wang et al ${ }^{8}$ reported recurrences after two curettage and autograft procedures in the ulna of a seven-year-old boy. They also suggested that the rate of recurrence reduces after the age of 18 years.

Extensive en-bloc resection is presumed to be curative, but such surgery is rarely required..$^{5}$ Operation has usually been recommended only in patients older than 15 years of age after repeated fractures with a rapidly progressive pathology. ${ }^{8,9,15}$ In our case there was an extensive and rapidly progressive recurrent lesion in a girl under ten years of age. The rate of progression of the condition accelerated after local resection, but a successful outcome was achieved by an extensive extraperiosteal resection and autografting procedure.

No benefits in any form have been received or will be received from a commercial party related directly or indirectly to the subject of this article.

\section{References}

1. Frangenheim P. Angeborene ostitis fibrosa als Ursache einer intrauterinen Unterschenkelfraktur. Arch Klin Chir 1921;117:22-9.

2. Kempson RL. Ossifying fibroma of long bones: a light and electron microscopic study. Arch Pathol 1966;82:218-33.

3. Johnson LC. Congenital pseudoarthrosis, adamantinoma of long bone and intracortical fibrous dysplasia of the tibia. J Bone Joint Surg [Am] 1972;54-A:1355.

4. Semian DW, Willis JB, Bove KE. Congenital fibrous defect of the tibia mimicking fibrous dysplasia. J Bone Joint Surg [Am] 1975;57-A:854-7.

5. Campanacci M. Osteofibrous dysplasia of long bones: a new clinical entity. Ital J Orthop Traumatol 1976;2:221-37.

6. Park YK, Unni K, McLeod RA, Pritchard DJ. Osteofibrous dysplasia: clinicopathologic study of 80 cases. Human Pathology 1993;24:1339-47.

7. Ozaki T, Hamada M, Taguchi K, et al. Polyostotic lesions compatible with osteofibrous dysplasia: a case report. Arch Orthop Trauma Surg 1993;113:46-8.

8. Wang JW, Shih CH, Chen WJ. Osteofibrous dysplasia (ossifying fibroma of long bones): a report of four cases and review of the literature. Clin Orthop 1992;278:235-43.

9. Campanacci M, Laus M. Osteofibrous dysplasia of the tibia and fibula. J Bone Joint Surg [Am] 1981;63-A:367-75.

10. Nakashima Y, Yamamuro T, Fujiwara Y, et al. Osteofibrous dysplasia (ossifying fibroma of long bones): a study of 12 cases. Cancer 1983;52:909-14.

11. Schajowicz F, Santini-Araujo E. Adamantinoma of the tibia masked by fibrous dysplasia: report of three cases. Clin Orthop 1989;238:294-301.

12. Kerr R. Radiologic case study: osteofibrous dysplasia. Orthopedics 1987;10:1082,1085-9.

13. Campbell CJ, Hawk T. A variant of fibrous dysplasia (osteofibrous dysplasia). J Bone Joint Surg [Am] 1982;64-A:231-6.

14. Schoenecker PL, Swanson K, Sheridan JJ. Ossifying fibroma of the tibia: report of a new case and review of the literature. J Bone Joint Surg [Am] 1981;63-A:483-8.

15. Markel SF. Ossifying fibroma of lone bone: its distinction from fibrous dysplasia and its association with admanatinoma of long bone. Am J Clin Pathol 1978;69:91-7. 